\title{
Atmospheric pressure and population density as super-factors influencing the transmission of coronavirus disease 2019 (COVID-19)
}

Wei Yan ( $\nabla$ yanwei@cug.edu.cn )

China University of Geosciences https://orcid.org/0000-0003-0271-3672

Muhammad Zohaib Nawaz

University of Agriculture, Faisalabad

Wenqian $\mathrm{Xu}$

China University of Geosciences

Zheng Jiang

China University of Geosciences

Weixiang Sun

China University of Geosciences

Jiayue Lai

China University of Geosciences

Yinghui Shao

China University of Geosciences

Wei Zhang

China University of Geosciences

Rui Zhang

Xiamen University

\section{Research Article}

Keywords: Coronavirus disease 2019, severe acute respiratory syndrome coronavirus 2, transmission, regression model, atmospheric pressure, population density

Posted Date: December 12th, 2020

DOI: https://doi.org/10.21203/rs.3.rs-93707/v3

License: (c) (1) This work is licensed under a Creative Commons Attribution 4.0 International License.

Read Full License 


\section{Abstract}

The novel severe acute respiratory syndrome coronavirus 2 (SARS-CoV-2), the etiologic agent of coronavirus disease (COVID-19), recently emerged and led to a global pandemic with enormous consequent losses to global health and economies. To date, more than 30 million cases have been reported globally and have affected almost every with varying degrees. Meteorological and nonmeteorological factors such as temperature, relative humidity, atmospheric pressure, population density, and latitude, are considered critical in virus transmission. To explore the correlation of environmental factors with the transmission of SARS-CoV-2 based on parameters including infection rate, effective reproduction number, and compound growth rate, we analyzed data of confirmed cases from 487 counties in the United States. We found a small impact of temperature and humidity on virus transmission, but observed a considerable positive influence of atmospheric pressure and population density on virus transmission. Geographic areas and seasons (autumn and winter), with exposure to higher atmospheric pressure, are more likely at higher risk of an outbreak. Social distancing and other measures could be effective strategies to combat COVID-19 outbreaks in densely populated areas. Additional studies are needed to explore the mechanisms underlying the relationship between meteorological parameters and transmission of SARS-CoV-2.

\section{Introduction}

The outbreak of coronavirus disease 2019 (COVID-19), caused by the novel severe acute respiratory syndrome coronavirus 2 (SARS-CoV-2), was declared a global pandemic by the World Health Organization on 11 March $2020^{1}$. And this pandemic has led to a global pandemic with enormous consequent losses to global health and economies ${ }^{2}$. By September 29, 2020, there were 33,417,386 confirmed cases of COVID-19, including 1,002,864 deaths, worldwide. The United States is the most affected country, with 7,150,824 confirmed cases and 205,107 deaths (Johns Hopkins Coronavirus Resource Center, https://coronavirus.jhu.edu). COVID-19 has touched nearly every country on the planet; however, its impact has varied widely. For example, the disease causes high mortality in Iran but lower mortality has been reported just across the border in Iraq, Bahrain, and Kuwait ${ }^{3}$. Scientists are trying to understand the puzzling behavior of SARS-CoV-2. Previous studies have found that various meteorological and non-meteorological factors, such as temperature and relative humidity, are critical in the spread of different viruses, including influenza virus ${ }^{4}$, respiratory syncytial virus (RSV) (GambaSanchez et al., 2016; Onozuka, 2015), severe acute respiratory coronavirus (SARS-CoV) ${ }^{5,6}$, and Middle East respiratory syndrome coronavirus (MERS-CoV) ${ }^{7}$. It is generally accepted that such environmental factors affect the stability of these pathogens and facilitate their spread. However, little is known about the underlying mechanisms involved in SARS-CoV-2 transmission.

Many countries around the world, such as China, have successfully controlled the outbreak using strategies such as effectively implementing social distancing and lockdowns, thereby minimizing humanto-human transmission of SARS-CoV-2. It is expected that subsequent outbreaks may occur during the 
autumn and winter seasons owing to the changes in meteorological conditions. Therefore, understanding the effect of meteorological factors on the spread of SARS-CoV-2 could help us devise effective strategies to prevent further outbreaks and spread of COVID-19. Many recent studies have reported a correlation between the COVID-19 pandemic and numerous meteorological variables ${ }^{8-12}$; however, the influence of meteorological factors on the spread of COVID-19 is not yet completely understood. In this study, we used data from 487 counties in the United States (US), collected from March 5, 2020 to May 1, 2020 , to determine the association of meteorological and non-meteorological factors with the early transmission of SARS-CoV-2 in the US. Our study provides a comprehensive analysis of factors associated with the spread of COVID-19.

\section{Results}

We calculated $R_{\text {proxy }} I R$, and $C G$ by analyzing the data from 487 counties in the US from March to May 2020. Data from 486, 482, and 303 counties $\left(R_{\text {proxy }}\right)$ and 487, 487, and 307 counties (IR and $C G$ ) were used to calculate the three parameters ( $R_{\text {proxy }}, I R$, and $C G$ ) for 17,24 , and 31 days after the 50 th confirmed case in each county, respectively. The $A T$ in these counties during this period ranged from $-3.43^{\circ} \mathrm{C}$ to $26.56^{\circ} \mathrm{C}$, and the $A A H$ varied from 2.56 to $24.18 \mathrm{~g} / \mathrm{m}^{3}$. Latitude ranged from $21.46^{\circ} \mathrm{N}$ to $64.81^{\circ} \mathrm{N}$, and $P D$ ranged from 1.2 to 11,103 inhabitants $/ \mathrm{mi}^{2}$ (Fig. 1 and S.1).

Potential relationships between early transmission of SARS-CoV-2 and environmental variables, including temperature, absolute humidity, atmospheric pressure, latitude, and $P D$ were explored using univariate regression analysis. In detail, $A A P$ and $P D$ were found to be key factors associated with virus transmission. Particularly, the average $R_{\text {proxy }}$ for 17 days showed a relationship with $\operatorname{AAP}\left(R^{2}=0.259\right.$, $\mathrm{P}<0.01)$ and $P D\left(R^{2}=0.200, \mathrm{P}<0.01\right)$; however, relatively small influences of $A T, A A H$, and latitude were observed (Table 1) (Fig. 2). Similarly, the $C G$ for 17 days showed a relationship with $A A P\left(R^{2}=0.176\right.$, $\mathrm{P}<0.01)$ and $\mathrm{PD}\left(R^{2}=0.325, \mathrm{P}=\mathrm{P}<0.01\right)$, but small influences of $A T, A A H$ and latitude were observed (Table S.1). The IR of COVID-19 displayed slight influences owing to all five environmental parameters (Table S.2). The overall results for 24 days also depicted a similar pattern but the influences of $A A P$ and $P D$ on $R_{\text {proxy }}$ and $C G$ were comparatively less than those for 17 days (Fig. S.2 and Tables S.3 and S.4). However, these patterns were not observed in the 31-day results (Tables S.6, S.7, and S.8).

The sMLR models showed that SARS-CoV-2 transmission could be explained by different combinations of parameters (Table 2). Specifically, latitude, $A A H, A A P$, and $P D$ explained $37.2 \%$ of the total variance for the 17-day $R_{\text {prxoy }}$ whereas latitude, $A T, A A P$, and $P D$ explained $41.0 \%$ of the total variance for the 17-day $C G$ (Table 2). In contrast, latitude, $A T, A A H, A A P$, and $P D$ explained only $20.5 \%$ of the total variance for the 17-day IR (Table 2). However, in the results for 24 and 31 days, the pattern of variance explained by these parameters was not followed (Tables S.9 and S.10).

\section{Discussion}


The present study was based on a relatively large dataset of the early transmission of COVID-19 in 487 US counties from March to May 2020. Our results suggest that both temperature and absolute humidity have a relatively small influence on the transmission of the virus that causes COVID-19, SARS-CoV-2. These findings are consistent with those of several recent studies conducted in China ${ }^{13}$ \{cmarchant:2020ki\} and Spain ${ }^{14}$. These results are also in good agreement with the fast-growing incidence of COVID-19 during the summer season, despite higher temperatures.

A novel finding of this study is the relationship between $A A P$ and SARS-CoV-2 transmission. This study showed that higher $A A P$ led to the rapid spread of COVID-19 during the early stage of the pandemic. Our results are consistent with a previous study conducted in Hubei Province of China, which demonstrated that the incidence of COVID-19 was positively correlated with $A A P^{15}$; however, our results are in contrast with other studies conducted in Japan ${ }^{16}$ and China ${ }^{17}$. Use of a larger dataset than in those previous studies and systematic approaches may lead to more robust conclusions. A positive correlation of atmospheric pressure with the spread of influenza virus ${ }^{18}$ and SARS-CoV ${ }^{19}$ has been found in previous studies. The underlying mechanism behind the relationship of $A A P$ with the transmission of COVID-19 is unclear. One explanation might be that increased $A A P$ may lead to an increase in the number of viruses per unit area when the human body expels respiratory viruses ${ }^{15}$. A positive correlation was observed between COVID-19 transmission and $P D$, consistent with previous study ${ }^{20}$, which also strengthens the notion that lockdown policies can help to flatten the epidemic curve ${ }^{21}$. The influence of $P D$ on the transmission of COVID-19 suggests that lockdown measures in cities with high $P D$, along with other measures including social distancing, isolation, and quarantine, are effective strategies to combat the transmission of COVID-19 in population-dense areas.

Our study is based on a comprehensive statistical analysis of a relatively large dataset. However, there are some limitations associated with this study. First, the observed $R_{\text {proxy }}$ across counties were estimated using available data, which may include incomplete records and this would add noise to the analysis results. Secondly, accurate estimates of SARS-CoV-2 infection are critical in our study; however, confirmed COVID-19 case counts in the US do not capture the total burden of the pandemic because testing has been primarily restricted to individuals with moderate to severe symptoms owing to limited test availability ${ }^{22}$. Third, our study included nearly 500 counties in the US; however, a much larger dataset including different countries and seasons worldwide is needed to better assess the relationships of meteorological and non-meteorological variables with virus transmission.

In conclusion, we found a minimal influence of temperature and absolute humidity on SARS-CoV-2 transmission. We, therefore, disagree with the expectation that outbreaks of COVID-19 are likely during the coming autumn and winter seasons owing to a fall in temperature. However, we observed a considerable positive influence of atmospheric pressure and population density on COVID-19 transmission in this study. Therefore, our results imply that increased atmospheric pressure during the coming autumn and winter may increase the risk of COVID-19 outbreaks in the northern hemisphere this year. The positive correlation of population density with COVID-19 transmission observed in this study 
shows that lockdown measures in population-dense cities are effective approaches to minimize disease transmission. The mechanisms underlying the relationship between meteorological parameters and the spread of COVID-19 need to be further explored. Additional studies are needed to better elucidate the role of meteorological and non-meteorological variables and to better forecast outbreak events during the ongoing COVID-19 pandemic.

\section{Materials And Methods}

\subsection{Study region and data}

We collected cumulative information of confirmed cases of COVID-19 in 487 US counties, reported by the Johns Hopkins Coronavirus Resource Center (https://github.com/CSSEGISandData/COVID-19) from March 5, 2020 to May 1, 2020. We used three parameters, including infection rate (IR), effective reproduction number $\left(R_{\text {proxy }}\right)$, and compound growth rate $(C G)$, to evaluate the spread of COVID-19.

$I R$ was calculated using the following equation:

$$
\operatorname{IR}(t)=\frac{C(t)}{N}
$$

where $C(t)$ denotes the cumulative number of confirmed cases per day $t$, and $N$ represents the total population of the county. IR during time interval $T$ was calculated using the following equation:

$$
I R^{(T)}=\frac{\sum_{t=1}^{T} I R(t)}{T}
$$

The effective reproduction number $\left(R_{\text {proxy }}\right)$ was used, as described in a study by Luo et al. ${ }^{13}$. Briefly, a proxy for the reproductive number $R$ in 5-day intervals was calculated using cumulative incidence data for each county. A proxy for $R, R_{\text {proxy }}$, indicates the occurrence of cases from time ( $\left.t\right)$ to time $(t+d)$ onto cases reported from time $(t+d)$ to time $(t+2 d)$, where $d$ is the calculated serial interval (i.e., the interval between successive cases in a series of disease transmissions). For multiple time points, $t$, values of $R_{\text {proxy }}(t, d)$ were obtained using the equation below:

$$
R_{\text {proxy }}(t, d)=\frac{C(t+2 d)-C(t+d)}{C(t+d)-C(t)}
$$

Taking $d$ as 5 , we estimate the $R_{\text {proxy }}$ of $D$ days (where $D \otimes 10$ ); for example, to calculate the $R_{\text {proxy }}$ of 17 days, we used the following formula:

$$
R^{(17)}=\frac{\sum_{t=1}^{7} R_{\text {proxy }}(t, 5)}{7}
$$


Compound growth rate (CG) was calculated using the following equation ${ }^{23}$ :

$$
C G=\left(\frac{C_{2}}{C_{1}}\right)^{\frac{1}{D}}
$$

Where $C_{1}$ represents the number of confirmed cases on the first day after the $50^{\text {th }}$ case, the $C_{2}$ represents the number of confirmed cases on the last day of the investigation period, and $D$ represents the duration (days) of the period.

Meteorological data, including temperature, relative humidity, and atmospheric pressure were collected from Reliable Prognosis (https://rp5.ru/Weather_in_the_world). We calculated the "absolute humidity" using temperature and relative humidity for each county with the following formula, which is an approximation of the Clausius-Clapeyron equation ${ }^{13}$ :

$$
A H=\frac{6.112 \cdot \mathrm{e}^{\frac{17.67243 .5}{2} \cdot R H \cdot 2.1674}}{T+273.15}
$$

where $\mathrm{AH}$ refers to absolute humidity, $\mathrm{T}$ is the temperature in Celsius, $\mathrm{RH}$ is the relative humidity (\%), and $e$ is the base of the natural log.

We calculated average temperature $(A T)$, average absolute humidity $(A A H)$, and average atmospheric pressure $(A A P)$ for 17, 24 and 31 days after the 50th confirmed case in each county. Population data at county level at the start of the year 2020 were obtained from the U.S. Census Bureau, and population density $(P D)$ was calculated (inhabitants $/ \mathrm{mi}^{2}$ ) for each county. To perform statistical analysis, we converted the population data logarithmically.

\subsection{Statistical and modeling analysis}

Statistical analysis was performed using the R statistical platform, v. 3.6.1 (The R Project for Statistical Computing, Vienna, Austria). First, univariate linear regression analysis was used to identify relationships between the measured environmental variables and $R_{\text {proxy }}, I R$, and $C G$ of COVID-19. $R$-squared values $\left(R^{2}\right)$ were calculated for the regression model to evaluate the percentage of variance in $R_{\text {proxy }}, I R$, and $C G$ of COVID-19 that could be explained by each environmental variable. Second, stepwise multiple linear regression (sMLR) models were developed. Model fit was assessed using $R^{2}$, and the Akaike information criterion was used to determine whether to add or remove variables during the stepwise procedure ${ }^{24}$. Before their use in SMLR, the data were standardized to their $z$-scores.

\section{Declarations}

Author contribution statement 
Wei Yan: Conceptualization, Methodology, Software, Writing-original draft, Supervision, Funding acquisition. Muhammad Zohaib Nawaz: Methodology, Software, Writing-review \& editing. Wenqian Xu: Software, Formal analysis. Zheng Jiang: Software, Investigation, Formal analysis. Weixiang Sun:

Software, Formal analysis. Jiayue Lai: Investigation. Yinghui Shao: Investigation. Wei Zhang:

Investigation. Rui Zhang: Conceptualization, Writing- review \& editing, Supervision, Funding acquisition.

\section{Declaration of competing interests}

The authors declare no conflict of interest.

\section{Acknowledgments}

The Fundamental Research Funds for the Central Universities (20720200027).

\section{References}

1. World Health Organization. WHO characterizes COVID-19 as a pandemic. (2020).

2. Jiao, N., Chen, F. \& Hou, Z. Combating climate change in a post-COVID-19 era. Sci Bull 1-3 (2020). doi:10.1016/j.scib.2020.08.017

3. World Health Organization. Coronavirus disease 2019 (COVID-19): situation report. (2020).

4. Ianevski, A. et al. Low Temperature and Low UV Indexes Correlated with Peaks of Influenza Virus Activity in Northern Europe during 2010-2018. Viruses 11, 207-10 (2019).

5. Cai, Q.-C. et al. Influence of meteorological factors and air pollution on the outbreak of severe acute respiratory syndrome. Public Health 121, 258-265 (2007).

6. Casanova, L. M., Jeon, S., Rutala, W. A., Weber, D. J. \& Sobsey, M. D. Effects of air temperature and relative humidity on coronavirus survival on surfaces. Appl Environ Microbio/ 76, 2712-2717 (2010).

7. Altamimi, A. \& Ahmed, A. E. Climate factors and incidence of Middle East respiratory syndrome coronavirus. J Infect Public Health 13, 704-708 (2020).

8. Auler, A. C., Cássaro, F. A. M., da Silva, V. O. \& Pires, L. F. Evidence that high temperatures and intermediate relative humidity might favor the spread of COVID-19 in tropical climate: A case study for the most affected Brazilian cities. Sci Total Environ 729, 139090 (2020).

9. Méndez-Arriaga, F. The temperature and regional climate effects on communitarian COVID-19 contagion in Mexico throughout phase 1. Sci Total Environ 735, 139560 (2020).

10. Prata, D. N., Rodrigues, W. \& Bermejo, P. H. Temperature significantly changes COVID-19 transmission in (sub)tropical cities of Brazil. Sci Total Environ 729, 138862 (2020).

11. Sajadi, M. M. et al. Temperature, Humidity and Latitude Analysis to Predict Potential Spread and Seasonality for COVID-19. SSRN Journal (2020). doi:10.2139/ssrn.3550308

12. Wu, Y. et al. Effects of temperature and humidity on the daily new cases and new deaths of COVID-19 in 166 countries. Sci Total Environ 729, 139051 (2020). 
13. Luo, W. et al. The role of absolute humidity on transmission rates of the COVID-19 outbreak. medRxiv 1-7 (2020). doi:10.1101/2020.02.12.20022467

14. Briz-Redón, Á. \& Serrano-Aroca, Á. A spatio-temporal analysis for exploring the effect of temperature on COVID-19 early evolution in Spain. Sci Total Environ 728, 138811 (2020).

15. Li, J. et al. Meteorological factors correlate with transmission of 2019-nCoV: Proof of incidence of novel coronavirus pneumonia in Hubei Province, China. medRxiv (2020). doi:10.1101/2020.04.01.20050526

16. Kuno, T., Yokoyama, Y., Matsushiro, T. \& Hari, Y. Higher Temperature, Pressure, and Ultraviolet Are Associated With Less COVID-19 Prevalence: Meta-Regression of Japanese Prefectural Data. Asia Pac J Public Health 1010539520947875 (2020). doi:10.1177/1010539520947875

17. Lin, S. et al. Region-specific air pollutants and meteorological parameters influence COVID-19: A study from mainland China. Ecotox Environ Safe 204, 111035 (2020).

18. Soebiyanto, R. P., Adimi, F. \& Kiang, R. K. Modeling and predicting seasonal influenza transmission in warm regions using climatological parameters. PLOS ONE 5, e9450 (2010).

19. Bi, P., Wang, J. \& Hiller, J. E. Weather: driving force behind the transmission of severe acute respiratory syndrome in China? Intern Med J 37, 550-554 (2007).

20. Lin, C. et al. A mechanism-based parameterisation scheme to investigate the association between transmission rate of COVID-19 and meteorological factors on plains in China. Sci Total Environ 737, 140348 (2020).

21. Tobías, A. Evaluation of the lockdowns for the SARS-CoV-2 epidemic in Italy and Spain after one month follow up. Sci. Total Environ. 725, 138539 (2020).

22. Wu, S. L. et al. Substantial underestimation of SARS-CoV-2 infection in the United States. Nat Comms 11, 4507 (2020).

23. Cobb, J. S. \& Seale, M. A. Examining the effect of social distancing on the compound growth rate of COVID-19 at the county level (United States) using statistical analyses and a random forest machine learning model. Public Health 185, 27-29 (2020).

24. Akaike, H. Markovian representation of stochastic processes and its application to the analysis of autoregressive moving average processes. Ann Inst Stat Math 26, 363-387 (1974).

\section{Tables}

Table 1 Statistics table for univariate linear and quadratic regression models of effective reproduction number ( $R_{\text {proxy }}$ ) of coronavirus disease 2019 (COVID-19) at county level for 17 days within the period under investigation. 


\begin{tabular}{|c|c|c|c|c|c|c|c|c|c|c|}
\hline & \multicolumn{10}{|c|}{ Dependent variable: } \\
\hline & & & & & & $R_{p r a x y}$ & & & & \\
\hline & (1) & (2) & (3) & (4) & (5) & (6) & (7) & (8) & (9) & (10) \\
\hline$\overline{A T}$ & $\begin{array}{l}-0.088^{*} \\
(0.045)\end{array}$ & $\begin{array}{l}-0.083^{*} \\
(0.048)\end{array}$ & & & & & & & & \\
\hline$A T^{2}$ & & $\begin{array}{l}-0.013 \\
(0.043)\end{array}$ & & & & & & & & \\
\hline Lat & & & $\begin{array}{c}0.060 \\
(0.045)\end{array}$ & $\begin{array}{c}0.066 \\
(0.045)\end{array}$ & & & & & & \\
\hline $\mathrm{Lat}^{2}$ & & & & $\begin{array}{l}-0.056^{* *} \\
(0.026)\end{array}$ & & & & & & \\
\hline$A A P$ & & & & & $\begin{array}{c}0.292^{* * *} \\
(0.044)\end{array}$ & $\begin{array}{c}0.238^{* * *} \\
(0.040)\end{array}$ & & & & \\
\hline$A A P^{2}$ & & & & & & $\begin{array}{c}0.254^{* * *} \\
(0.024)\end{array}$ & & & & \\
\hline$A A H$ & & & & & & & $\begin{array}{l}-0.080^{*} \\
(0.045)\end{array}$ & $\begin{array}{l}-0.060 \\
(0.064)\end{array}$ & & \\
\hline$A A H^{2}$ & & & & & & & & $\begin{array}{l}-0.020 \\
(0.045)\end{array}$ & & \\
\hline $\log (P D)$ & & & & & & & & & $\begin{array}{c}0.407^{* * *} \\
(0.042)\end{array}$ & $\begin{array}{c}0.434^{* * *} \\
(0.041)\end{array}$ \\
\hline $\log \left(P D^{2}\right)$ & & & & & & & & & & $\begin{array}{c}0.119^{* * *} \\
(0.026)\end{array}$ \\
\hline Constant & -0.000 & 0.013 & -0.000 & 0.056 & -0.000 & $-0.253^{* * *}$ & -0.000 & 0.020 & 0.000 & $-0.118^{* *}$ \\
\hline & $(0.045)$ & $(0.063)$ & $(0.045)$ & $(0.052)$ & $(0.044)$ & $(0.046)$ & $(0.045)$ & $(0.064)$ & $(0.042)$ & $(0.048)$ \\
\hline Observations & 484 & 484 & 484 & 484 & 484 & 484 & 484 & 484 & 484 & 484 \\
\hline $\mathrm{R}^{2}$ & 0.008 & 0.008 & 0.004 & 0.013 & 0.085 & 0.259 & 0.006 & 0.007 & 0.166 & 0.200 \\
\hline Adjusted $\mathrm{R}^{2}$ & 0.006 & 0.004 & 0.002 & 0.009 & 0.083 & 0.256 & 0.004 & 0.003 & 0.164 & 0.196 \\
\hline Residual Std. Error & $0.997(\mathrm{df}=482)$ & $\begin{array}{c}0.998(\mathrm{df}= \\
481)\end{array}$ & $\begin{array}{c}0.999(\mathrm{df}= \\
482)\end{array}$ & $0.995(\mathrm{df}=481)$ & $0.957(\mathrm{df}=482)$ & $0.862(\mathrm{df}=481)$ & $0.998(\mathrm{df}=482)$ & $\begin{array}{c}0.999(\mathrm{df}= \\
481)\end{array}$ & $0.914(\mathrm{df}=482)$ & $0.897(\mathrm{df}=481)$ \\
\hline F Statistic & $\begin{array}{c}3.781^{*}(\mathrm{df}=1 \\
482)\end{array}$ & $\begin{array}{c}1.934(\mathrm{df}=2 ; \\
481)\end{array}$ & $\begin{array}{c}1.758(\mathrm{df}=1 \\
482)\end{array}$ & $\begin{array}{c}3.274^{* *}(\mathrm{df}=2 \\
481)\end{array}$ & $\begin{array}{c}44.911^{* * *}(\mathrm{df}=1 \\
482)\end{array}$ & $\begin{array}{c}84.235^{* * *}(\mathrm{df}=2 \\
481)\end{array}$ & $\begin{array}{c}3.085^{*}(\mathrm{df}=1 \\
482)\end{array}$ & $\begin{array}{c}1.640(\mathrm{df}=2 ; \\
481)\end{array}$ & $\begin{array}{c}95.708^{* * *}(\mathrm{df}=1 \\
482)\end{array}$ & $\begin{array}{c}59.944^{* * *}(\mathrm{df}=2 \\
481)\end{array}$ \\
\hline
\end{tabular}

Table 2 Statistics table for stepwise multiple regression models of average (a) effective reproduction number ( $R_{\text {proxy }}$ ), (b) compound growth $(C G)$, and (c) infection rate (IR) of coronavirus disease 2019 (COVID-19) at the county level for 17 days within the period under investigation. 


\begin{tabular}{|c|c|c|c|}
\hline & \multicolumn{3}{|c|}{ Dependent variable: } \\
\hline & $\begin{array}{l}R_{\text {proxy }} \\
\text { (1) }\end{array}$ & $\begin{array}{l}C G \\
(2)\end{array}$ & $\begin{array}{l}I R \\
(3)\end{array}$ \\
\hline$A T^{2}$ & & $\begin{array}{c}0.127^{* * *} \\
(0.046)\end{array}$ & $\begin{array}{l}0.102^{*} \\
(0.054)\end{array}$ \\
\hline Lat & & $\begin{array}{l}0.070^{*} \\
(0.042)\end{array}$ & $\begin{array}{l}0.250^{* *} \\
(0.099)\end{array}$ \\
\hline$L a t^{2}$ & $\begin{array}{c}-0.069^{* * *} \\
(0.022)\end{array}$ & $\begin{array}{c}-0.088^{* * *} \\
(0.028)\end{array}$ & $\begin{array}{c}-0.152^{* * *} \\
(0.036)\end{array}$ \\
\hline$A A P$ & $\begin{array}{c}0.198^{* * *} \\
(0.039)\end{array}$ & $\begin{array}{c}0.124^{* * *} \\
(0.038)\end{array}$ & \\
\hline$A A P^{2}$ & $\begin{array}{c}0.223^{* * *} \\
(0.022)\end{array}$ & $\begin{array}{c}0.142^{* * *} \\
(0.023)\end{array}$ & \\
\hline$A A H$ & & & $\begin{array}{l}0.257^{* *} \\
(0.104)\end{array}$ \\
\hline $\log (P D)$ & $\begin{array}{c}0.297^{* * *} \\
(0.039)\end{array}$ & $\begin{array}{c}0.471^{* * *} \\
(0.038)\end{array}$ & $\begin{array}{c}-0.218^{* * *} \\
(0.042)\end{array}$ \\
\hline $\log \left(P D^{2}\right)$ & $\begin{array}{c}0.078^{* * *} \\
(0.024)\end{array}$ & $\begin{array}{c}0.117^{* * *} \\
(0.023)\end{array}$ & $\begin{array}{c}0.239^{* * *} \\
(0.027)\end{array}$ \\
\hline Constant & $\begin{array}{c}-0.231^{* * *} \\
(0.051)\end{array}$ & $\begin{array}{c}-0.297^{* * *} \\
(0.056)\end{array}$ & $\begin{array}{c}-0.188^{* * *} \\
(0.065)\end{array}$ \\
\hline Observations & 484 & 487 & 487 \\
\hline $\mathrm{R}^{2}$ & 0.372 & 0.410 & 0.205 \\
\hline Adjusted $\mathrm{R}^{2}$ & 0.366 & 0.402 & 0.195 \\
\hline Residual Std. Error & $0.796(\mathrm{df}=478)$ & $0.773(\mathrm{df}=479)$ & $0.897(\mathrm{df}=480)$ \\
\hline F Statistic & \multicolumn{3}{|c|}{$56.743^{* * *}(\mathrm{df}=5 ; 478) 47.627^{* * *}(\mathrm{df}=7 ; 479) 20.601^{* * *}(\mathrm{df}=6 ; 480)$} \\
\hline
\end{tabular}

\section{Figures}
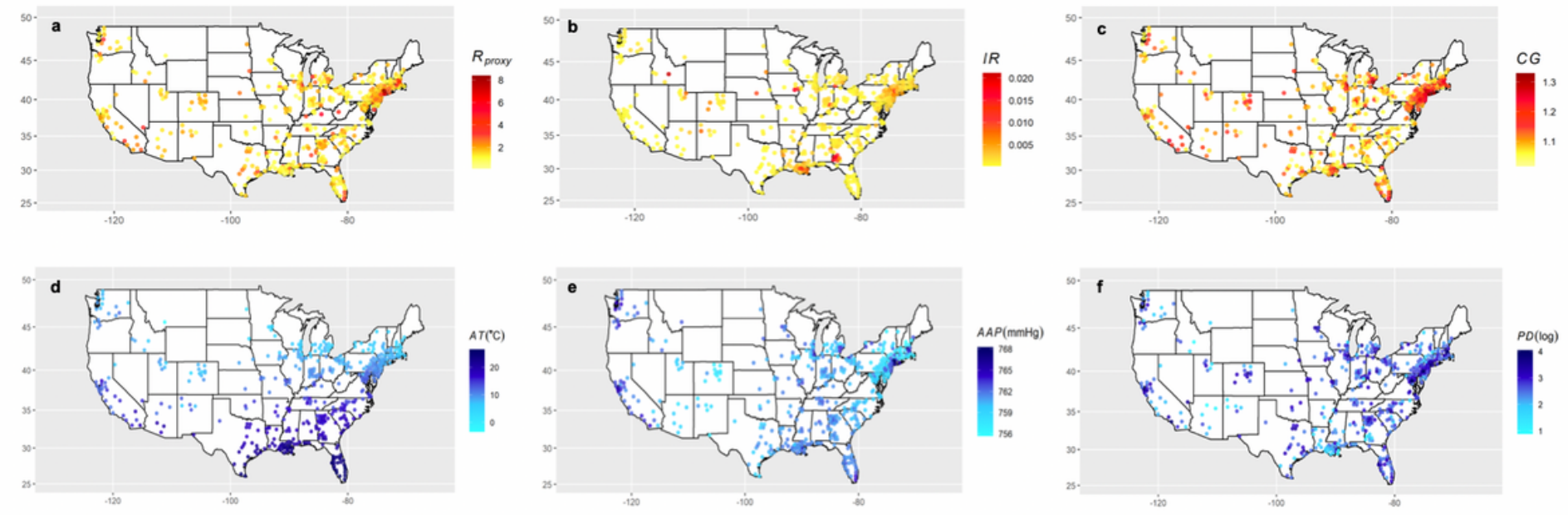

Figure 1 
Average (a) effective reproduction number (Rproxy), (b) infection rate (IR), and (c) compound growth (CG) of coronavirus disease 2019 (COVID-19) and average (d) temperature (AT) and (e) atmospheric pressure (AAP) at county level for 17 days within the period under investigation. (f) Population density (PD).
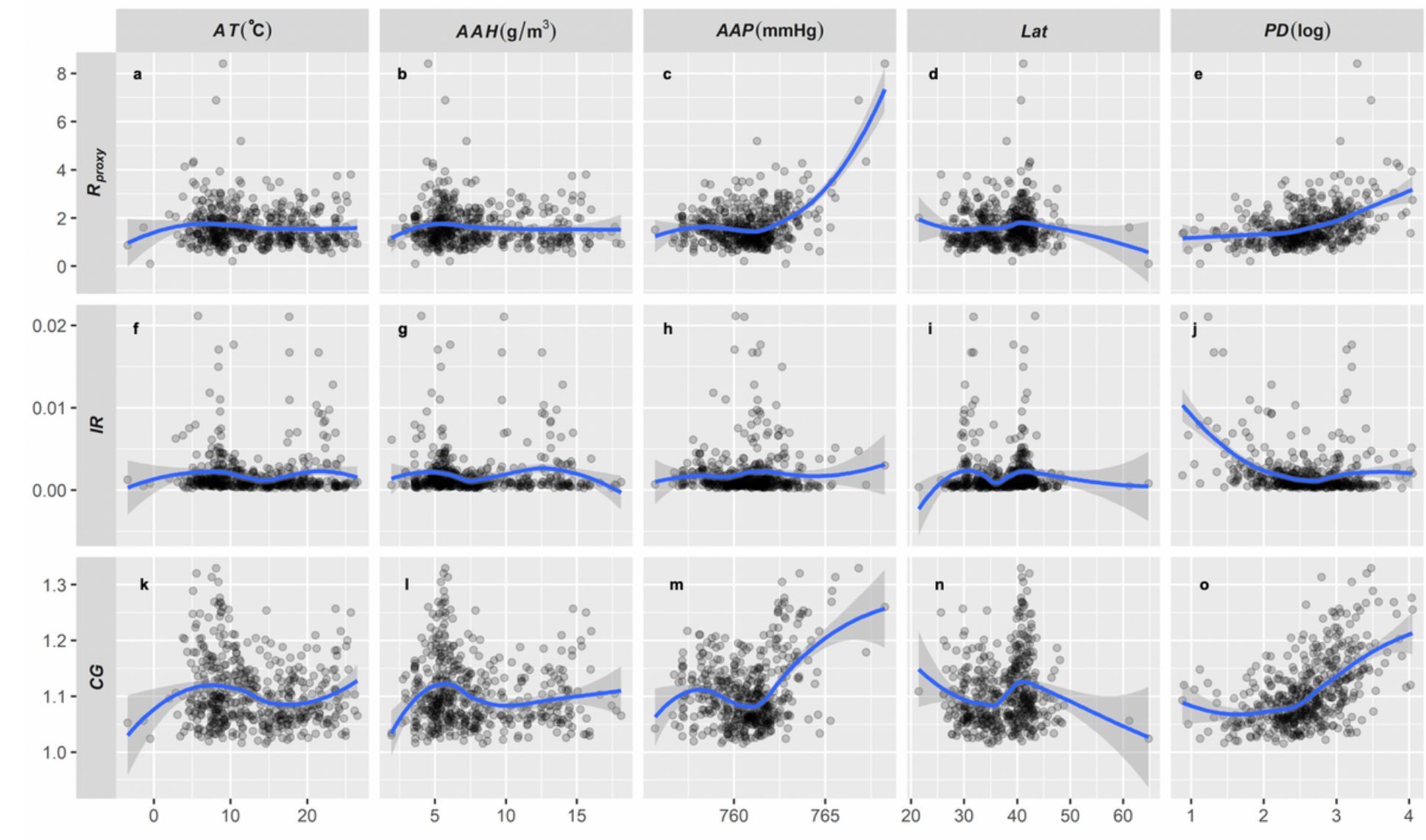

Figure 2

The 17-day average effective reproduction number (Rproxy), infection rate (IR), and compound growth (CG) of coronavirus disease 2019 (COVID-19) plotted as a function of average temperature (AT)(a, f, k), average absolute humidity $(A A H)(b, j, i)$, average atmospheric pressure $(A A P)(c, h, m)$, latitude $(d, i, n)$, and population density $(P D)(e, j, o)$ at the county level.

\section{Supplementary Files}

This is a list of supplementary files associated with this preprint. Click to download.

- SARS2SI20201019.docx 